\title{
Extremely efficient direct third harmonic generation in thin nanostructured films of $\mathrm{ZnO}$
}

 \\ ${ }^{\mathrm{a}}$ Department of Physics, University of Wisconsin - Milwaukee, P. O. Box 413, Milwaukee, WI \\ 53201, USA

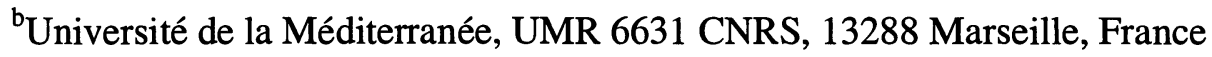

\begin{abstract}
We report a very efficient nonlinear optical conversion in thin films of wide band-gap materials. Very high conversion efficiency to the third harmonic radiation is achieved for an unamplified femtosecond $\mathrm{Cr}^{4+}$ :forsterite laser in a sub-micron-thick film of a nanocrystalline $\mathrm{ZnO}$ pulsed-laser deposited on a fused silica substrate. Both the nonlinear optical coefficient and the coherence length are measured for a film composed of $10-\mathrm{nm}$ nanoclusters.
\end{abstract}

Keywords: Nonlinear optics, femtosecond, nanocrystal, $\mathrm{ZnO}$, third-harmonic generation

\section{INTRODUCTION}

Wide band-gap semiconductors recently have attracted interest for their applications in solid-state electronics and optics [1-2]. In particular, zinc oxide ( $\mathrm{ZnO}$ ) is a II-VI semiconductor with a wide band-gap of 3.37-eV, and a direct structure at room temperature [3]. It is especially attractive because of the recent demonstrations of its applicability as a perspective material for near-UV emitters [4-6] and ultrafast UV light modulators [7-8]. On the other hand, there is a significant demand for thin-film nonlinear optical materials, which can be easily integrated into an optoelectronic device [1-2, 9-10]. It has been recently pointed out that $\mathrm{ZnO}$ films have a strong nonlinear second-order optical susceptibility, $\chi^{(2)}$, and the efficient second-harmonic has been generated in thin films of $\mathrm{ZnO}$ [11-12]. However, most of the nonlinear optical devices are using higher order susceptibility, $\chi^{(3)}$ [13]. II-VI semiconductors fall in the group of materials with a very high nonlinear refractive index, $\mathrm{n}_{2}$, which has been measured for $\mathrm{ZnO}$ to be $2.3^{\prime} 10^{-12}$ esu (for $\lambda=1.064 \mu \mathrm{m}$ ) [14], or $\chi^{(3)}=1.2 \cdot 10^{-13}$ esu, making it one of the possible materials for nonlinear optical device applications. Furthermore, nonresonant nonlinear optical susceptibilities of these materials may be significantly affected by the small size of nanocrystals [15-16]. Here we report on the efficient third-harmonic generation in thin films of $\mathrm{ZnO}$ leading to direct generation of UV light from an unamplified near-IR radiation of femtosecond $\mathrm{Cr}^{4+}$ :forsterite laser $(1200-1300 \mathrm{~nm})$.

Third-harmonic generation is a nonlinear process, in which incident high intensity laser radiation at the frequency $\omega$, interacting with a nonlinear medium, results in the generation of an additional spectral component at the frequency $3 \omega$. For a tight focused beam it can be generated only on the interface between two media [17-20]. Assuming that third harmonic is generated in a very thin film (i.e. film thickness is much less than a confocal parameter of the laser beam, $b$, the power of the generated third harmonic is given by the following formulae [17]:

$I_{3 \omega}=\frac{2304 \pi^{6}\left|\chi^{(3)}\right|}{n_{1}^{3} n_{3} \lambda_{1}^{4} c^{2}} I_{\omega}^{3} \frac{d^{2} \sin ^{2}(\Delta k d / 2)}{(\Delta k d / 2)^{2}}$,

where $n_{I}$ and $n_{3}$ - are the refractive indices of the film at the fundamental frequency and the frequency of the third harmonic, respectively, $\lambda_{I}$ - is the wavelength of the fundamental radiation, $d$ - is the film thickness, $\chi^{(3)}$ - is the nonlinear optical susceptibility of the film, $\Delta k$ - is the wavevector mismatch between the fundamental and thirdharmonic waves in a film, $\underline{I}_{\underline{\omega}}$ - is the power of the incident radiation. For extremely thin films, i.e. when the film thickness is much less than the coherence length:

\footnotetext{
*Email: yakovlev@uwm.edu; Phone: +1-414-229-3978; Fax: +1-414-229-5589
} 


$$
l_{c o h}=\frac{2 \pi}{\Delta k},
$$

the intensity of the third harmonic becomes proportion to the square of the film thickness.

It is clear, that in order to achieve high conversion efficiency, one has to increase the incident intensity of the laser and use materials with the highest possible nonlinear optical coefficient $\chi^{(3)}$. An increase of the incident laser intensity above a certain threshold level will damage the material; however, wide band-gap materials have shown resistance to the laser-induced damage up to the level of $>1 \mathrm{~J} / \mathrm{cm}^{2}$ [21]. $\mathrm{ZnO}$ is also transparent to the third harmonic of 1200-1300 nm radiation, thus minimizing the probability of its damage by the intense third harmonic beam. The use of femtosecond optical pulses $\left(100 \mathrm{fs}=10^{-13} \mathrm{~s}\right)$ results in the intensity level of $>10^{13} \mathrm{~W} / \mathrm{cm}^{2}$. Competing nonlinear optical effects such as self-phase modulation, which typically lead to destruction of useful nonlinear optical mixing processes, are significantly reduced in thin films, because of a limited distance of light interaction with a nonlinear material.

\section{SAMPLE PREPARATION AND EXPERIMENTAL SET-UP}

Our sample is grown on a thin (1-mm-thick) fused silica substrate by a pulsed laser deposition. The nanostructured $\mathrm{ZnO}$ films have been prepared in a mixed $\mathrm{O}_{2}$-He background gas atmosphere by conventional UV pulsed laser (ArF

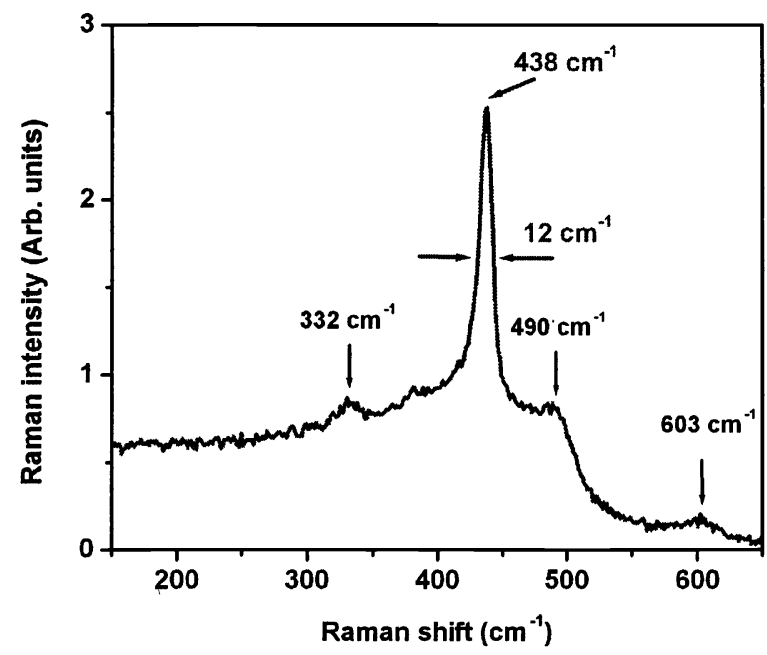

Figure 1. Raman spectrum of a nanocrystalline film of $\mathrm{ZnO}$.

laser, $\lambda=193 \mathrm{~nm}$ ) ablation [22]. First, $\mathrm{ZnO}$ nanoclusters were synthesized in the gas phase, during laser induced plume expansion and then, relatively could, stabilized, clusters were deposited on fused silica substrate. The substrate temperature was $380^{\circ} \mathrm{C}$. Resulting nanostructured films have oriented structure, with $\mathrm{C}$ axis perpendicular to the substrate. The cluster size distribution is narrow and can be described by Gaussian function with a maximum at $10 \mathrm{~nm}$ and a half width of $3 \mathrm{~nm}$ [23]. The films, prepared with the optimized substrate temperature and gas partial pressures, have an intense UV excitonic luminescence and a very weak defect-related band. The film thickness varies along the surface of a substrate and, thus, allows measurements of film parameters as a function of film thickness. The maximum film thickness is slightly over $1 \mu \mathrm{m}$.

First of all, we confirm the homogeneity of nanoclusters along the surface of the film. We use a conventional Raman microscopy with a typical spatial resolution of about $1.5 \mu \mathrm{m}$ and spectral resolution $<3 \mathrm{~cm}^{-1}$ to measure the Raman spectrum at different points on the film's surface. A typical spectrum is shown in Figure 1, where the background signal from a substrate is numerically subtracted. The major feature of this spectrum is a strong $438 \mathrm{~cm}^{-1}$ line, which has a bandwidth of about $12 \mathrm{~cm}^{-1}$, corresponding to nanoparticles of $\mathrm{ZnO}$ with a size diameter of $10 \mathrm{~nm}$ [24]. This 
line bandwidth remains the same over the whole surface of the film as we would expect from our other independent measurements [21].

The experimental set-up we use for our experiments is shown in Figure 2. The laser system we use is a home-built femtosecond $\mathrm{Cr}^{4+}$ :forsterite laser. By extending the length of the laser cavity we have recently demonstrated up to 17 nJ per pulse at the repetition rate of $27 \mathrm{MHz}$ [25]. The pulse duration is typically 30-40 fs and the center wavelength around $1250 \mathrm{~nm}$. The output of the laser is taken from the dispersive end of the cavity and is sent through the pair of prisms to compensate for the dispersion of the output coupler and the focusing lens. We use an aspheric lens (ThorLabs, Inc.) with a numerical aperture of 0.55 and a focal length of $4.5 \mathrm{~mm}$ to focus the laser radiation into the sample. The third harmonic light is then collected from the back of the sample by using a 0.4-N.A. microscope objective and is redirected either into spectrometer with an attached CCD detector for spectral measurements or to a power meter. A calibrated filter is used to select the spectral radiation around $420 \mathrm{~nm}$ (the wavelength of the third harmonic of our fundamental radiation).

\section{EXPERIMENTAL RESULTS}

First of all, we move the focal plane of the incoming beam on the surface of $\mathrm{ZnO}$ film. It can be easily accomplished by simultaneous recording of both third- and second- harmonic of the fundamental in the reflection geometry. We

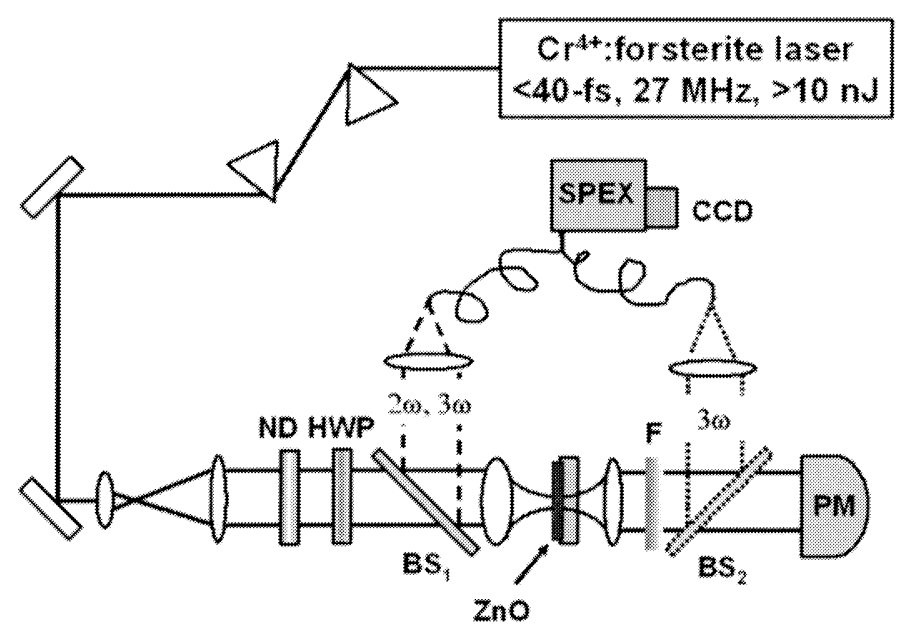

Figure 2. Experimental set-up for the third-harmonic generation. $\mathrm{BS}_{1,2}$ - are dichroic beamsplitters, ND - neutral density filter wheel, $\mathrm{F}$ - blue transmitting, near-IR absorbing filter, PM- power meter, HWP - half-wave plate.

find that the spatial position for the maximum of reflected both third and second harmonics corresponds to the position of the maximum of the transmitted third harmonic signal. By scanning the sample through the focal plane of the laser beam we can determine the confocal parameter of the beam and derive the beam waist [19-20]. From these measurements we can calculate the beam waist of the laser beam to be $(1.8 \pm 0.2) \mu \mathrm{m}$ (measured at the full-width half-maximum). The intensity of the third harmonic generated from the fused silica/air interface is at least 4 orders of magnitude weaker than one generated from the $\mathrm{ZnO} /$ air interface. We also confirm (see Figure 3) that the intensity of the third harmonic is proportional to the third power of the incoming intensity up to the maximum intensity used in our experiments ( $350 \mathrm{~mW}$ of the incident power, corresponding to $13 \mathrm{~nJ}$ per pulse, which leads to the energy density of $0.5 \mathrm{~J} / \mathrm{cm}^{2}$ ). While the cascaded third harmonic generation through the second harmonic generation and consequent mixing of the generated radiation with the fundamental beam is possible, the efficiency of it for disoriented (in the plane of the substrate) film of $\mathrm{ZnO}$ is negligible. The later fact is experimentally confirmed by direct measurements of the second harmonic power. It appears to be many orders of magnitude weaker than the power of the third harmonic. There is no any degradation of the third harmonic intensity with time that makes us believe that the damage threshold for our $\mathrm{ZnO}$ film is higher than the maximum energy level used in our experiments. We also confirm that the spectrum of the third harmonic (see inset of the Figure 3) remains independent on the incident power level. The Fourier transformation of this spectrum corresponds to the transform- 
limited pulse duration of about 20-fs. Since the collection optics is not optimized for this particular spectral region no attempt has been made to compress and measure the pulse duration of the generated UV pulses.

We find that the efficiency of the third harmonic generated at the maximum power level is surprisingly high. The energy of the generated third harmonic depends on the position on the film and has strong thickness dependence. We measure up to $1.5 \mathrm{~mW}$ of the average power at the maximum incident power. When the appropriate losses on

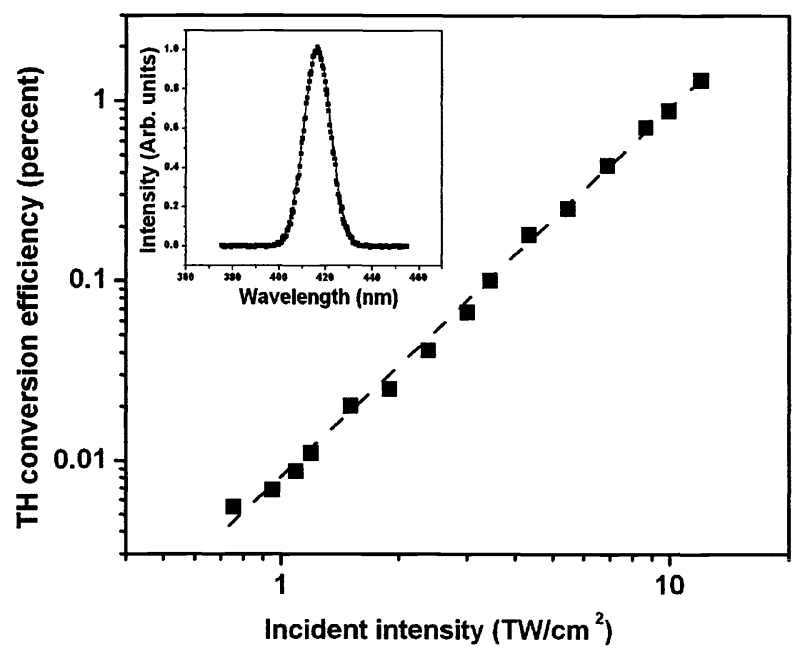

Figure 3. Experimentally measured conversion efficiency, $P_{3 \omega} / P_{\omega}$, where $P_{3 \omega}$ is the power of the generated third harmonic, and $P_{\omega}$ - is the incident power of the fundamental beam. Dashed line shows a theoretically predicted quadratic dependence. On the inset - experimentally measured spectrum (squares) of the third harmonic at the maximum available input power. Solid line shows a theoretically predicted spectrum of the third harmonic, derived from the spectrum of the incident radiation.

reflections and filter absorption are taken into account we get a little more than $4.5 \mathrm{~mW}$ of generated UV light or $1.3 \%$ conversion efficiency into the coherent third harmonic. For a relatively thin film (approximately $200 \pm 30 \mathrm{~nm}$ thick) we can employ the Eq. (1) to calculate the value of $\chi^{(3)}$ for measured conversion efficiency. We get the value of $\chi^{(3)}=(1.4 \pm 0.7) \cdot 10^{-12}$ esu, assuming the incident intensity of $(1.2 \pm 0.2) \cdot 10^{13} \mathrm{~W} / \mathrm{cm}^{2}$. This value is significantly higher than earlier reported value of $\chi^{(3)}$, derived from $\mathrm{n}_{2}$ measurements [14], which we attribute to the nanosized structure of the film. This reduced dimensionality of the film also plays a significant role in the enhancement of the second order susceptibility $\chi^{(2)}$ in thin films of $\mathrm{ZnO}$ [12]. Recently significant ( 500 times) enhancement of $\mathrm{n}_{2}$ with respect to the bulk value has been observed for polymer-capped $\mathrm{ZnO}$ nanocrystals with an estimated average size of $4 \mathrm{~nm}$ [16]. We also have tried an epitaxially grown film of $\mathrm{ZnO}$, but the energy of the third harmonic generated from these films is more than an order of magnitude smaller than one from the nanocrystalline film of the same thickness, the fact that confirms our hypothesis on the nanostructured nature of observed very efficient nonlinear optical conversion from our original films.

It is a very important question to understand the dependence of the generated third harmonic on the thickness of this film. We scan the focal point of the laser beam across the surface of the film measuring simultaneously the film thickness and energy of the generated third harmonic. These data are shown as crosses in Figure 4. It is clearly seen the modulated dependence of the third harmonic on the film thickness. In order to describe it we have to include the effects of the finite coherence length for nonlinear optical interaction in the film. Since no data are available for the refractive index of $\mathrm{ZnO}$ nanocrystals we use the data for the bulk material [26] to calculate the coherence length for the third harmonic generation process in the case of the incident light propagating along the $\mathrm{C}$ axis of the crystal. We obtain the value of coherence length of $l_{c o h}{ }^{\text {calc }}=900 \mathrm{~nm}$, which is in a reasonably good agreement with a value of $l_{c o h}{ }^{e x p}=900 \mathrm{~nm}$, obtained by fitting experimental data in Figure 4 with a function described in Eq.(1). We note that the energy of generated third harmonic does not drop to zero, as one would expect. However, these films are not 
considered to be homogeneous by all means, so a deviation of their optical properties is likely to occur. The effects of very tight focusing and light scattering in these films also may affect the phase-matching process. Nevertheless, we can conclude that under given experimental conditions we have, indeed, obtained the maximum conversion efficiency in these films, which is of the order of percent.

We also note that no damage or any degradation to the output power of the third harmonic has been observed for the highest possible conversion efficiencies. This measured maximum conversion efficiency is favorably compared to one for cascaded third harmonic generation in conventional nonlinear crystals [27], and the proposed nonlinear

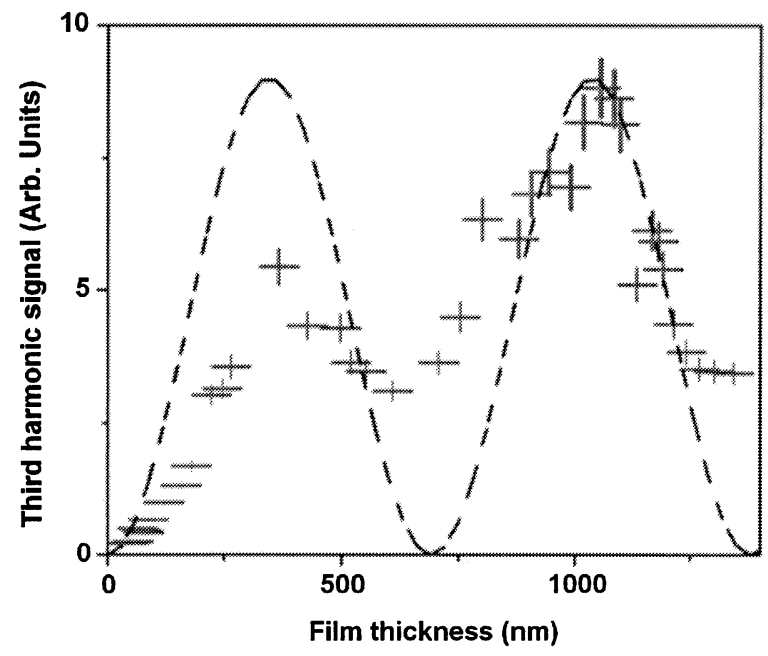

Figure 4. Experimentally measured dependence of the third harmonic signal on the film thickness (crosses). Experimental errors of the measured values of film thickness and power of the third harmonic are shown as the size of crosses.

Dotted line is a fit using Eq. (1).

optical device based on a thin-film nanostructured $\mathrm{ZnO}$ film is much easier to implement since no specific angular adjustments are needed. It is also important that the deposited film is isotropic, and the efficiency of the third harmonic is independent of the incoming polarization, which is confirmed by rotating the polarization of the incident beam using a half-wave plate, i.e. nonlinear optical device based on the thin film of $\mathrm{ZnO}$ can generate efficient third harmonic for any linear polarization of incident radiation.

It is also interesting to scale our measurements down to a single nanoparticle (i.e. to the volume of about $(10 \mathrm{~nm})^{3}$. Once again, assuming that the nonlinearity does not drop dramatically with the size, we obtain under the above focusing conditions the efficiency of the third harmonic to be about $10^{9}$ photons/s. Partial support of these estimations has been recently demonstrated with near field optical measurements using $800-\mathrm{nm}$ excitation light [28].

Finally, we also try to compare our results on $\mathrm{ZnO}$ with the third harmonic generated from thin disordered films of $\mathrm{GaN}$ and $\mathrm{TiO}_{2}$ of about the same thickness produced by different techniques on sapphire and fused silica substrates. While both these materials are considered to have significant optical nonlinearity [14], we conclude that the third harmonic signal from nanostructured $\mathrm{ZnO}$ at least 2 orders of magnitude stronger than one from $\mathrm{GaN}$ and $\mathrm{TiO}_{2}$ films of the same thickness. These observations indicate that more studies are needed to understand the physical mechanisms of significant enhancement of third-order nonlinear susceptibility of $\mathrm{ZnO}$ nanocrystals [15-16]. We are currently preparing thin films with smaller average size of nanocrystals, for which we expect an even stronger thirdharmonic signal and plan on reporting of these results in a later communication.

\section{CONCLUSION}

In summary, we have demonstrated that $\mathrm{ZnO}$ thin film has a very strong third-order nonlinear optical coefficient, making it possible to directly generate third harmonic of ultrashort pulses with efficiency of the order of percent. 
High-efficiency of nonlinear optical conversion makes is possible to use thin films of nanostructured $\mathrm{ZnO}$ for thirdorder autocorrelation measurements of weak pulses [29] and characterization of single nanocrystals.

\section{ACKNOWLEDGEMENTS}

The authors would like to acknowledge support and helpful discussions with Dr. A. Sklyarov (The Advanced Analysis Facility, University of Wisconsin - Milwaukee). This work is partially supported by the NSF Grant 9984225, and NIH Grant R21RR16282.

\section{REFERENCES}

1. M. M. Fejer, “Nonlinear-optical frequency-conversion”, Physics Today 47, 25-32 (1994).

2. H. Shen, M. Wraback, J. Pamulapati, S. Liang, C. Gorla, and Y. Lu, "Properties of epitaxial ZnO thin films for GaN and related applications", MRS Internet J. Nitride Semicond. Res. 4S1, G3.60 (1999).

3. Y. Ohta, T. Haga, and Y. Abe, “Crystallographic features of ZnO single crystals “, Jpn. J. Appl. Phys. 36, L1040-L1042 (1997).

4. D. M. Bagnall, Y. F. Chen, Z. Zhu, T. Yao, M. Y. Shen, and T. Goto, "High temperature excitonic stimulated emission from ZnO epitaxial layers “, Appl. Phys. Lett. 73, 1038-1040 (1998).

5. Z. K. Tang, G. K. L. Wong, P. Yu, M. Kawasaki, A. Ohtomo, H. Koinuma, and Y. Segawa, "Room-temperature ultraviolet laser emission from self-assembled $\mathrm{ZnO}$ microcrystallite thin films", Appl. Phys. Lett. 70, 2230-2232 (1997).

6. H. Cao, J. Y. Xu, E. W. Seelig, and R. P. H. Chang, "Microlaser made of disordered media “, Appl. Phys. Lett. 76, 2997-2999 (2000).

7. M. S. Wu, T. Shiosaki, and A. Kawabata, "Electro-optic diffraction modulation in ZnO film on sapphire", IEEE J. Quant, Electron. 25, 252-256 (1989).

8. W. Wraback, H. Shen, S. Liang, C. R. Gorla, and Y. Lu, "High contrast, ultrafast optically addressed ultraviolet light modulator based upon optical anisotropy in $\mathrm{ZnO}$ films grown on R-plane sapphire ", Appl. Phys. Lett. 74, 507-509 (1999).

9. P. M. Lundquist, M. Jurich, J. F. Wang, H. Zhou, T. J. Marks, and G. K. Wong, "Electro-optical characterization of poled-polymer films in transmission “, Appl. Phys. Lett. 69, 901-903 (1996).

10. W. P. Lin, P. M. Lundquist, G. K. Wong, E. D. Rippert, and J. B. Ketterson, "2-nd-order optical nonlinearities of radio-frequency sputter-deposited AIN thin-films", Appl. Phys. Lett. 63, 2875-2877 (1993).

11. H. Cao, J. Y. Wu, H. C. Ong, J. Y. Dai, and R. P. H. Chang, Appl. Phys. Lett. 73, 572-574 (1998).

12. G. Wang, G. T. Kiehne, G. K. L. Wong, J. B. Ketterson, X. Liu, and R. P. H. Chang, "Second harmonic generation in laser ablated zinc oxide thin films", Appl. Phys. Lett. 80, 401-403 (2002).

13. G. P. Agrawal, Nonlinear Fiber Optics, Academic Press, New York, 2001.

14. R. Adair, L. L. Chase, and S. A. Payne, "Nonlinear refractive-index of optical crystals", Phys. Rev. B39, $3337-$ 3350 (1989).

15. G. Banfi, V. Degiorgio, and D. Ricard, "Nonlinear optical properties of semiconductor nanocrystals ", $A d v$. Phys. 47, 447-510 (1998).

16. L. Guo, S. Yang, C. Yang, P. Yu, J. Wang, W. Ge, and G. K. L. Wong, "Highly monodisperse polymer-capped ZnO nanoparticles: Preparation and optical properties “, Appl. Phys. Lett. 75, 2901-2903 (2000).

17. J. F. Reintjes, Nonlinear optical parametric processes in liquids and gases, Academic Press, Orlando, 1984.

18. T. Y. F. Tsang, "Optical 3-rd harmonic generation at interfaces”, Phys. Rev. A52, 4116-4125 (1995).

19. Y. Barad, H. Eisenberg, M. Horowitz, and Y. Silberberg, "Nonlinear scanning laser microscopy by third harmonic generation", Appl. Phys. Lett. 70, 922-924 (1997). 
20. J. M. Schins, T. Schrama, J. Squier, G. Brakenhoff, and M. Muller, "Determination of material properties by use of third-harmonic generation microscopy “, J. Opt. Soc. Am. B19, 1627-1634 (2002).

21. W. Kautek, J. Kruger, M. Lenzner, S. Srtania, C. Spielmann, and F. Krausz, "Laser ablation of dielectrics with pulse durations between 20 fs and 3 ps “, Appl. Phys. Lett. 69, 3146-3148 (1996).

22. W. Marine, L. Patrone, B. Luk'yanchuk, and M. Snetis, "Strategy of nanocluster and nanostructure synthesis by conventional pulsed laser ablation”, Appl. Surf. Sci. 154, 345-352 (2000).

23. I. Ozerov, D. Nelson, A. V. Bulgakov, W. Marine, and M. Sentis, "Synthesis and laser processing of $\mathrm{ZnO}$ nanocrystalline thin films “, Appl. Surf. Sci. 212, 349-352 (2003).

24. Y. Du, M. S. Zhang, J. Hong, Y. Shen, Q. Chen, and Z. Yin, "Structural and optical properties of nanophase zinc oxide ", Appl. Phys. A 76, 171-176 (2003).

25. V. Shcheslavskiy, V. V. Yakovlev, and A. Ivanov, "High-energy self-starting femtosecond $\mathrm{Cr}^{4+}: \mathrm{Mg}_{2} \mathrm{SiO}_{4}$ oscillator operating at a low repetition rate", Opt. Lett. 26, 1999-2001 (2001).

26. CRC handbook of laser science and technology: Optical Materials, M. J. Weber Ed., CRC Press, 1997.

27. X. Mu, X. Gu, M. Makarov, Y. J. Ding, L. Wang, J. Wei, and Y. Liu, "Third-harmonic generation by cascading second-order nonlinear processes in a cerium-doped $\mathrm{KTiOPO}_{4}$ crystal”, Opt. Lett. 25, 117-119 (2000).

28. J. C. Johnson, H. Q. Yan, R. D. Schaller, P. B. Peterson, P. D. Yang, and R. J. Saykally, "Near-field imaging of nonlinear optical mixing in single zinc oxide nanowires ", Nano Letters 2, 279-283 (2002).

29. D. Meshulach, Y. Barad, and Y. Silberberg, "Measurement of ultrashort optical pulses by third-harmonic generation ", J. Opt. Soc. Am. B14, 2122-2125 (1997). 